\title{
La loi de 2015 sur l'islam en Autriche
}

Francis Messner

\section{OpenEdition}

Journals

Édition électronique

URL : http://journals.openedition.org/rdr/965

DOI $: 10.4000 /$ rdr.965

ISSN : 2534-7462

\section{Éditeur}

Presses universitaires de Strasbourg

\section{Édition imprimée}

Date de publication : 8 novembre 2016

Pagination : 177-183

ISBN : $978-2-86820-959-7$

ISSN : 2493-8637

\section{Référence électronique}

Francis Messner, "La loi de 2015 sur l'islam en Autriche », Revue du droit des religions [En ligne], 2 2016, mis en ligne le 11 février 2020, consulté le 19 novembre 2020. URL : http:// journals.openedition.org/rdr/965; DOI : https://doi.org/10.4000/rdr.965

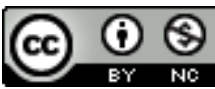

La revue du droit des religions est mise à disposition selon les termes de la Creative Commons Attribution - Pas d'Utilisation Commerciale 4.0 International - CC BY-NC 4.0. 


\section{LA LOI DE 2015 SUR L'ISLAM EN AUTRICHE}

\section{Francis MESSNER}

Université de Strasbourg / CNRS, Droit, Religion, Entreprise et Société (DRES)

$\mathrm{L}$ e droit des relations entre l'État et les religions en Autriche distingue les sociétés religieuses reconnues qui sont des collectivités de droit public, les communautés religieuses enregistrées qui sont des personnes juridiques de droit privé et les religions organisées dans le cadre du droit commun d'association. Les sociétés religieuses sont reconnues grâce à divers mécanismes juridiques : concordat pour l'Église catholique, lois spéciales ou procédure fixée par la loi sur la reconnaissance de 1874. Leur statut est fixé par des normes constitutionnelles. La société religieuse islamique a été reconnue par une loi spéciale en 1912, remplacée par une nouvelle loi en 2015.

Pays majoritairement catholique depuis la Contre-Réforme ${ }^{1}$, l'Autriche reste attachée au régime des cultes instauré au XIX ${ }^{e}$ siècle et au statut privilégié de l'Église catholique, qui reçoit notamment des subventions de l'État en contrepartie des biens confisqués sous le régime nazi ${ }^{2}$. Ainsi, seul 0,89\% de l'électorat s'est prononcé lors d'un référendum d'initiative populaire organisé en 2013 portant sur une stricte séparation des Églises et de l'État et sur l'abolition des privilèges des Églises ${ }^{3}$.

1. $73 \%$ de catholiques, $5 \%$ de protestants, $4,30 \%$ de musulmans et seulement $12 \%$ de sans confession selon le recensement de 2001 (la France comptabilise $43 \%$ de sans confession en 2016 selon l'enquête Sociovision pour Le Monde des religions).

2. Convention du 23 juin 1960 entre le Saint-Siège et la République d'Autriche portant réglementation des relations patrimoniales.

3. Référendum contre les privilèges des Églises (Volksbegehren gegen Kirchenprivilegien), 15 au 22 avril 2013. 
La liberté de religion et des convictions philosophiques se rapportant à l'immanence est garantie de manière extensive par la loi fondamentale de l'État de 1867 (StGG), l'article 63 du Traité d'État de St-Germain-en-Laye de 1919 et par l'article 9 de la CEDH.

Les relations entre les religions et l'État ${ }^{4}$ sont adossées au principe de neutralité - l'État est séparé des religions - et à la coopération entre les autorités publiques et les représentants des corporations religieuses, mise en œeuvre par le biais du statut des cultes octroyé par l'État aux religions. L'article 15 StGG fixe ainsi le statut des cultes reconnus qui sont dotés de la personnalité juridique de droit public. Ils gèrent et administrent de manière autonome leurs propres affaires en restant, comme toute autre collectivité, soumis aux lois générales. Ils sont traités de manière égale par l'État, leurs biens nécessaires au culte, à la charité et à l'éducation sont protégés. Le droit à un enseignement religieux confessionnel dans les écoles publiques est garanti par l'article $17 \mathrm{StGG}$ à toutes les sociétés religieuses reconnues ${ }^{5}$.

La procédure de reconnaissance conférée par un règlement du chancelier fédéral (Verordnung) est fixée par la loi du 20 mai 1874 relative à la reconnaissance légale des sociétés religieuses ${ }^{6}$. Aux termes de l'article $1^{\text {er }}$ de ce texte, la reconnaissance en tant que société religieuse peut être accordée aux membres d'une religion sous réserve que leur doctrine religieuse, leur liturgie, leur constitution et la dénomination choisie ne contiennent rien de contraire à la loi ou de moralement choquant et que l'existence d'au moins une communauté cultuelle ${ }^{7}$ créée conformément à cette loi soit assurée. La société religieuse demanderesse doit en outre faire état d'un nombre d'adhérents correspondant à $0,2 \%$ au moins de la population autrichienne ${ }^{8}$. Les sociétés religieuses déjà « reconnues » historiquement sous différentes formes avant 1874 sont organisées par des lois spéciales. Il s'agit de l'Église catho-

4. V. Potz R., «Église et État en Autriche », in Robbers G. (dir.), État et Églises dans l'Union européenne, Baden Baden, Nomos, $2^{e}$ éd. 2008, p. 417 : http://www.uni-trier.de [consulté le 3 juin 2016]

5. Un cours de substitution portant sur l'éthique est suivi par les élèves qui n'appartiennent pas à une société religieuse reconnue.

6. Sont actuellement reconnus aux termes de la loi de 1874, l'Église vieille-catholique, l'Église méthodiste, l'Église de Jésus-Christ des Saints des derniers jours (mormons), l'Église arménienne-apostolique, l'Église néo-apostolique d'Autriche, la religion bouddhiste, l'Église syrienne orthodoxe et les Témoins de Jéhovah.

7. Il s'agit des communautés de base, la paroisse par exemple pour l'Église catholique et pour l'Église protestante.

8. Loi fédérale du 9 janvier 1998 relative à la personnalité juridique des communautés confessionnelles. 
lique dont le statut est actuellement et pour l'essentiel régi par le Concordat de 1933 ratifié en mai 1934, de l'Église protestante rassemblant luthériens et réformés relevant d'une loi de 1961, des orthodoxes (loi de 1967) et orthodoxes orientaux (loi de 2003), des juifs (loi de 1890) et des musulmans (loi de 1912, puis de 2015).

Par ailleurs, une loi du 9 janvier 1998 fixe les conditions de l'acquisition de la personnalité juridique en tant que société religieuse enregistrée pour les religions ${ }^{9}$ ne bénéficiant pas de la reconnaissance. L'enregistrement au registre des communautés religieuses a pour effet de créer une personne juridique de droit privé qui a droit à l'appellation de « communauté confessionnelle enregistrée par l'État », ce qui est pour le moins une reconnaissance symbolique. La communauté concernée doit, en vue de son enregistrement, fournir la preuve du nombre de ses adhérents fixé à 300 au minimum, et ses statuts comprenant le nom, les modes d'entrée et de sortie de la communauté (affiliation et désaffiliation), les modes de désignation des organes dirigeants, son organisation territoriale, ses moyens financiers et une présentation des doctrines et de la pratique religieuses. Le ministre fédéral de l'Enseignement et des Affaires culturelles doit refuser l'enregistrement lorsque la doctrine et son application sont susceptibles de constituer des troubles à l'ordre public ${ }^{10}$.

La loi sur l'islam (Islamgesetz) de 1912 était le résultat d'une politique religieuse pragmatique visant à intégrer toutes les populations et religions dans l'Empire. Elle a été adoptée suite à l'annexion de la Bosnie-Herzégovine par l'Empire d'Autriche-Hongrie en 1908, année charnière qui marque le début de l'effondrement de l'Empire ottoman. Cette loi spéciale ${ }^{11}$ de reconnaissance s'appliquait dans un premier temps à la Reichshälfte, c'est-àdire l'Autriche-Hongrie, et fut étendue dans un second temps à tout l'Empire pour y permettre l'exercice du culte musulman de rite hanafite, les cultes non reconnus n'étant pas autorisés à exercer publiquement leur religion. La chute de l'Empire austro-hongrois en 1918 n'a cependant pas permis de mettre

9. La loi de 1998 ne s’applique pas aux groupements philosophiques ou communautés idéologiques (athées, humanistes...).

10. Huit confessions religieuses sont actuellement enregistrées en Autriche dont les hindouistes et l'Église de l'unification de Moon. L'enregistrement de l'Église de scientologie a été refusé par l'administration.

11. La reconnaissance par le biais de la loi de 1874 n'a pas été rendue possible dans la mesure où l'islam ne remplissait pas toutes les conditions fixées par ce texte : absence d'organes représentatifs et de textes fixant son organisation. 
en œuvre cette loi qui s'appliquera pour la première fois en $1979^{12}$ pour la reconnaissance de l'Islamische Glaubensgemeinschaft in Österreich (IGGiÖ). Depuis une décision de la Cour constitutionnelle de 1987, la reconnaissance d'une organisation musulmane n'est plus subordonnée à l'appartenance au rite hanafite ${ }^{13}$ qui est une des écoles du sunnisme ${ }^{14}$. Aux termes d'une autre décision de la Cour de $2010^{15}$, d'autres groupements musulmans peuvent également demander la reconnaissance en application de la loi de 1874 , ce qui est le cas des alévis, reconnus depuis 2013, alors que les "vieux alévis » sont constitués en communautés confessionnelles enregistrées depuis 2015. Les communautés chiites ne remplissent quant à elles ni les conditions de la reconnaissance, ni celles de l'enregistrement.

L'Islamgestz de 1912 ne permettant pas d'atteindre l'objectif visé par les pouvoirs publics d'organiser de manière unifiée l'ensemble des groupements musulmans, elle a été remplacée par une nouvelle loi adoptée en $2015^{16}$. L'Islamgesetz de 2015 définit les droits et devoirs de la " communauté religieuse islamique en Autriche » (Islamische Glaubensgemeischaft in Österreich, art. 9 à 15) et de la « communauté religieuse alévie en Autriche " (Islamische Alevitische Glaubensgemeinschat in Österreich, art. 16 à 22). L'article premier de cette loi de 2015 renvoie à l'article 15 StGG $^{17}$ qui fixe le statut des sociétés religieuses reconnues, collectivités de droit public qui gèrent leurs affaires internes de manière autonome. L'article 2 précise que les sociétés religieuses musulmanes, leurs communautés locales et leurs membres pris individuellement ont le devoir de respecter les lois. La doctrine et les règlements propres à l'islam ne peuvent se substituer au droit autrichien, le principe de liberté d'organisation des cultes inscrit dans la loi constitutionnelle fédérale n'entraînant pas une concurrence entre le droit étatique et les droits et disciplines religieux.

Les sociétés religieuses musulmanes acquièrent la reconnaissance, et par voie de conséquence la personnalité juridique de droit public, par règlement

12. Décision du ministre fédéral de l'Éducation et de l'Art du 2 mai 1979.

13. VfSlg 11.574/1987.

14. Cette décision a entraîné une réorganisation de l'IGGiÖ : règlement du ministre fédéral de l'Éducation, de l'Art et du Sport du 2 août 1988.

15. VfGH 1. 12. 2010, B 1214/09.

16. Bundesgesetz über die äußeren Rechtsverhältnisse islamischer Religionsgesellschaften - Islamgesetz 2015, BGBl. I Nr. 39/2015.

17. Art. 15. : « Toute église ou communauté religieuse reconnue par la loi a droit à l'exercice public et collectif de sa religion, règle et administre ses affaires intérieures librement, garde la propriété et la jouissance de ses établissements, fondations et fonds destinés au culte, à l'enseignement et à la bienfaisance, tout en restant, comme toute société, soumise aux lois générales de l'État». 
du chancelier fédéral. Leur création est subordonnée à la dissolution des associations cultuelles qui les représentaient jusqu'alors. Cette disposition souligne la volonté des pouvoirs publics autrichiens d'unifier l'organisation de l'islam en évitant un éclatement institutionnel, même si l'administration ne peut pas s'opposer à une demande de reconnaissance émanant d'un groupement cultuel en se fondant sur cet argument ${ }^{18}$. La reconnaissance d'une nouvelle société religieuse musulmane est subordonnée dans la loi de 2015 aux conditions suivantes (art. 4) : capacité d'exister dans la durée avec un nombre de membres s'élevant au moins à $0,2 \%$ de la population, autonomie financière, "attitude positive envers la société et l'État », absence de tensions avec d'autres sociétés religieuses reconnues ou non reconnues. La reconnaissance est refusée (art. 5) selon des dispositions similaires à celles fixées par la loi de 1998 pour les sociétés religieuses enregistrées : protection dans une société démocratique de la sécurité publique, de l'ordre, de la santé ou de la morale publics, protection des droits et libertés d'autrui, en particulier dans le cas d'entrave au développement psychique de jeunes adultes, de violation de l'intégrité psychique et dans le cas d'utilisation de méthodes psychothérapeutiques, notamment à des fins prosélytes. Le ministre doit en outre mettre fin à la reconnaissance lorsqu'une des conditions de la reconnaissance énumérées n'est pas ou plus remplie, ou si persiste un comportement contraire au statut malgré la mise en demeure d'y mettre un terme.

Le dossier en vue de la création d'une collectivité religieuse musulmane de droit public doit notamment comprendre une présentation de la doctrine et des textes fondateurs de cette religion (art. 6), exigence qui n'est pas inscrite dans la loi de 1874 qui fixe dans son article 6 le contenu des statuts. L'article 6 (2) de la loi de 2015 impose par ailleurs aux membres de la société religieuse résidant en Autriche de financer l'ensemble des besoins religieux et cultuels, alors même que la loi de 1939 mettant en place un impôt d'Église (Kirchenbeitrag) au profit des sociétés religieuses protestante, catholique et vieille-catholique n'est pas étendue aux musulmans. En d'autres termes, le financement par des institutions ou des États étrangers est interdit. Cette disposition a notamment pour but de mettre fin au détachement en Autriche d'imams fonctionnaires de la Direction des affaires religieuses de Turquie (Diyanet) : les imams doivent désormais être formés et recrutés en Autriche. D'une manière générale, l'autonomie financière est une condition sine qua non de la reconnaissance et cette obligation de moyens financiers suffisants figure dans la procédure de reconnaissance déterminée par l'article 5 de la

18. VfGH 1. 12. 2010, B 1214/09. 
loi de 1874. Dans l'optique du législateur, le soutien économique d'un État étranger induit une dépendance qui ne garantit pas l'existence d'une société religieuse sur une longue durée. La nouveauté est que l'Islamgesetz de 2015 écarte certains modes de financement.

L'article 24 de la loi de 2015 consacré à la collaboration entre les sociétés religieuses et l'État prévoit la création d'une faculté de théologie musulmane ${ }^{19}$ au sein de l'université publique de Vienne ${ }^{20}$. Pourvue de six postes d'enseignants-chercheurs, la faculté dispensera un enseignement et développera des recherches avec une attention particulière portée à la formation des imams et des professeurs de religion musulmane dans les établissements d'enseignement publics et privés (écoles, collèges, lycées). Elle mettra en œuvre deux programmes séparés, l'un destiné aux imams sunnites (IGGiÖ), l'autre aux imams alévis. La création d'un enseignement de théologie au sein d'une université publique résulte d'un équilibre entre la liberté de recherche et d'enseignement des enseignants-chercheurs et la liberté de religion collective des sociétés religieuses ${ }^{21}$, dans le cadre du principe d'autonomie des universités $^{22}$. Cet équilibre difficile à trouver est cependant nécessaire. L'absence d'un accord avec la société religieuse concernée peut être interprétée comme une absence de légitimité qui ferait obstacle à l'inscription des futurs imams à la faculté de théologie musulmane. Inversement, la transmission efficace de l'islam dans le contexte contemporain, en tant que facteur d'intégration des musulmans dans une société sécularisée, sur la base du sens critique et d'une approche rationnelle des traditions musulmanes, suppose une intégration fonctionnelle dans l'université publique.

Les articles 12 et 19 de la loi de 2015 autorisent les sociétés religieuses musulmanes sunnites et alévies à organiser la fabrication de produits alimentaires conformément aux règles propres à l'islam ${ }^{23}$. Ils prévoient également que

19. L'État fédéral autrichien finance depuis 1998 un Institut de pédagogie religieuse privé (IRPA) créé pour prendre en charge la formation des enseignants de religion dans les établissements d'enseignement publics et privés. Aux termes de la loi sur les études universitaires de 1999, l'ensemble des instituts de pédagogie ont été transformés en établissements d'enseignement supérieurs de pédagogie intégrés dans le système public. Deux chaires de pédagogie religieuse musulmane ont été créées avant 2015 dans les universités de Vienne et d'Innsbruck.

20. L'université de Vienne comprend déjà une faculté de théologie catholique et une faculté de théologie protestante.

21. La nomination des enseignants de théologie musulmane de l'université publique se fera après consultation des autorités des sociétés religieuses musulmanes et alévies.

22. Article 81c de la loi fédérale constitutionnelle d'Autriche.

23. V. Grabenwarter Ch. et Gartner-Muller B., « Das österreichische Islamgesetz 2015 und ihre rechtliche Genese », Kirche \& Recht, 1/2015, p. 47-73. 
les pouvoirs publics prendront en considération les prescriptions alimentaires des musulmans dans l'armée, les prisons, les hôpitaux et les établissements d'enseignement publics. Enfin, les jours fériés du calendrier musulman et musulman alévi sont protégés par l'État (art. 13 et 20) et des cimetières ou parties de cimetières sont instaurés avec l'accord des autorités religieuses (art. 15 et 22).

Les statuts nationaux des cultes en Europe sont marqués par l'histoire comme en témoigne la loi autrichienne sur l'islam de 1912 qui n'est pas sans évoquer le joséphisme visant à asseoir une politique de contrôle et d'intégration des groupements religieux. On assiste au cours de la période récente à une récidive générée par les évolutions du paysage religieux : la loi sur les communautés religieuses enregistrées de 1998 procède de la même démarche. La nouvelle loi sur l'islam n'atteint pas l'objectif initial visé par le pouvoir politique qui était de créer une représentation unifiée. La quasitotalité des musulmans relève cependant de ce texte qui instaure toutefois des organisations séparées pour les musulmans et les musulmans alévis. La volonté de créer un islam d'Autriche (Islam österreichischer Prägung) a généré sous certains aspects une différence de traitement par rapport aux autres cultes reconnus. La loi insiste sur la suprématie du droit étatique sur la discipline et les doctrines religieuses et prohibe les subventions des États et institutions étrangères. Cette dernière mesure qui vise à instaurer un corps d'imams formés et rémunérés en Autriche est assortie de la création d'une faculté de théologie à l'université de Vienne. Les politiques religieuses en Autriche bénéficient de moyens à hauteur des objectifs visés. 